Bio - grafia. Escritos sobre la Biología y su Enseñanza. ISSN 2027

Edición Extraordinaria. p.p. 621 - 635

Memorias del VIII Encuentro Nacional de Experiencias en Enseñanza de la Biología y la Educación Ambiental. III Congreso Nacional de Investigación en Enseñanza de la Biología.

\title{
Diseño de una matriz de análisis para temas ambientales en proyectos de ciencias naturales
}

\author{
Ana Ilse Benavides Lahnstein ${ }^{1}$, Renteria Yei ${ }^{2}$, Alma Adrianna Gómez Galindo ${ }^{2}$, Rocío \\ Guadalupe Balderas Robledo²
}
${ }^{1}$ CSSME, School of Education, University of Leeds, UK; ${ }^{2}$ Unidad Monterrey - Cinvestav, México edaibl@leeds.ac.uk; yrenteria@cinvestav.mx; agomez@cinvetav.mx; rbalderas@cinvestav.mx

RESUMEN. En ésta investigación estudiamos los contenidos ambientales de dos proyectos de ciencias naturales del libro de texto para el sexto año de educación primaria en México. El trabajo por proyectos, el cual fue formalmente introducido al sistema de educación básica en el último proceso de reforma, es presentado como una estrategia para consolidar el conocimiento de los estudiantes para alcanzar los aprendizajes esperados. Nuestro estudio se guío por dos preguntas principales: ¿Satisfacen los contenidos ambientales de los proyectos seleccionados los aprendizajes esperados del currículo de ciencias naturales para sexto grado? ¿Son estos contenidos ambientales pertinentes con las problemáticas actuales de la educación ambiental para la sustentabilidad? Para contestarlas realizamos una investigación documental en documentos oficiales y diseñamos un instrumento para la codificación y el análisis del contenido de los proyectos. Los resultados se discutieron en tres unidades de análisis de acuerdo a los tres niveles de complejidad planteados en la matriz. Identificamos en los proyectos contenidos ambientales básicos con referencia a los aprendizajes esperados. Consideramos que el éxito potencial del contenido ambiental depende de las competencias de los docentes y de los estudiantes para ponerlos en práctica efectivamente.

Palabras clave: educación ambiental, educación primaria, trabajo por proyectos, educación para la sustentabilidad 
Bio - grafia. Escritos sobre la Biología y su Enseñanza. ISSN 2027

Edición Extraordinaria. p.p. 621 - 635

Memorias del VIII Encuentro Nacional de Experiencias en Enseñanza de la Biología y la Educación Ambiental. III Congreso Nacional de Investigación en Enseñanza de la Biología.

\begin{abstract}
.
In this investigation we studied the environmental content of two projects within the natural sciences textbook for primary school education in Mexico. Working by projects, which was formally introduced to the basic education system in the last reform process, is presented as a strategy to consolidate students' knowledge in order to meet expected learning outcomes. Our study was guided by to two main questions: do the environmental content in the selected projects satisfy the expected learning outcomes of the year six natural sciences curriculum? Are the environmental contents in accordance with the general concerns of environmental education for sustainability? To answer these questions we conducted a documentary investigation of official documents and designed an instrument to codify and analyse the content of the projects. We identified within the projects basic environmental content with reference to the expected learning outcomes. Furthermore potential success of environmental content depends on teachers and students' competences to put them into practice effectively.
\end{abstract}

Keywords: environmental education, primary school education, project based learning, education for sustainability

\title{
INTRODUCCIÓN
}

En este escrito comunicaremos el proceso y los resultados que obtuvimos al implementar una matriz de análisis de contenido ambiental a un par de proyectos del libro de texto de ciencias naturales de sexto año de primaria en México. Los contenidos de educación ambiental para la sustentabilidad en los documentos oficiales y libros de texto de educación primaria Mexicana carecen de dimensiones de complejidad, aportes teóricos para la resolución de problemas ambientales y métodos de evaluación de los contenidos. A raíz de esto nos preguntamos ¿Satisfacen estos contenidos ambientales los aprendizajes esperados del currículo de ciencias naturales para sexto grado? ¿Tienen los proyectos analizados contenido ambiental pertinente con las problemáticas actuales de la educación ambiental para la sustentabilidad? 
Bio - grafia. Escritos sobre la Biología y su Enseñanza. ISSN 2027

Edición Extraordinaria. p.p. 621 - 635

Memorias del VIII Encuentro Nacional de Experiencias en Enseñanza de la Biología y la Educación Ambiental. III Congreso Nacional de Investigación en Enseñanza de la Biología.

Los documentos oficiales no proveen un instrumento para analizar la pertinencia de los contenidos ambientales, pero hay estándares curriculares que ofrecen una guía de expectativas. La mayor parte de los puntos contenidos en los estándares curriculares para ciencias naturales en sexto año de primaria abordan la dimensión ambiental; aunque, sin el apoyo de contenidos ambientales pertinentes y explícitos, alcanzar estos estándares es una tarea desafiante para el docente y el estudiante. Consideramos, junto a otros autores (SEMARNAT, 2006; Peza Hernández, 2014), que la formación docente, el currículo y las practicas didácticas sugeridas para la educación ambiental para la sustentabilidad aún presentan áreas de oportunidad.

\section{MARCO TEÓRICO}

En la práctica educativa los saberes teóricos de la educación ambiental toman forma de "contenidos ambientales", aunque no todos los contenidos de un currículo para la educación ambiental pueden alcanzar ésta clasificación. En el contexto de la pedagogía, Benítez Esquivel (2009) menciona que un contenido ambiental "es un tema o problema que permite mirar, cuestionar, aprender, comprender y reflexionar acerca de un sistema específico - un grupo escolar, una comunidad - y sobre las múltiples relaciones que conforman su ambiente" (p.47-48). Complementamos lo anterior con el trabajo de Ángel Maya (1996), considerado miembro de la Escuela de Pensamiento Ambiental Latinoamericano, quien plantea la relación entre sistema cultural y sistema natural también conocido como ecosistema. Aunado a la cultura él los define como dos subsistemas abiertos que se relacionan con sus elementos y forman parte de un sistema mayor llamado sistema natural. Maya propone tres niveles de interacción: del ecosistema hacia la cultura (desde las formas de adaptación); de la cultura hacia el ecosistema, es decir los impactos (con factores como la población, su paradigma tecnológico, su organización social, económica, familiar y su mundo simbólico) y finalmente; del ecosistema nuevamente hacia la cultura. Para Maya el ambiente no consiste exclusivamente en el medio que nos rodea y la suma de las especies o a las poblaciones biológicas en él contenidas. El ambiente representa además una categoría social constituida por comportamientos, valores y saberes actuales a lo largo de la historia. 
Bio - grafia. Escritos sobre la Biología y su Enseñanza. ISSN 2027

Edición Extraordinaria. p.p. 621 - 635

Memorias del VIII Encuentro Nacional de Experiencias en Enseñanza de la Biología y la Educación Ambiental. III Congreso Nacional de Investigación en Enseñanza de la Biología.

\section{METODOLOGÍA}

Utilizamos la investigación documental, aquí recuperamos el plan de estudios 2011 que se encuentra vigente para la educación básica y educación primaria y el programa de estudios de ciencias naturales para sexto año de primaria y libro de texto de la misma asignatura (ciclo escolar 2014-2015). Para la fase de análisis desarrollamos una matriz de análisis para contenidos ambientales.

Marco contextual

En este trabajo analizamos dos proyectos que se ubican y describen a continuación.

En los programas de estudios ciencias naturales (asignatura cursada del tercer al sexto año de educación primaria en México) algunos de los propósitos de estudio apuntan al cuidado del ambiente, conocer las interacciones del ser humano con el medio y propuestas en torno al consumo sustentable (SEP, 2011a:83). El caso es similar para geografía, formación cívica y ética e historia, sugiriendo un tratamiento transversal del área (SEP, 2011a). En los programas y libros de texto, ciencias naturales está organizada por cinco ámbitos o campos de conocimiento. Éstos se dividen en cinco bloques; cada uno se imparte durante un bimestre del ciclo escolar. Como se muestra en la figura 1, cada bloque (excepto el quinto) contiene tres temas, subtemas y un proyecto que se propone desarrollar en las últimas dos semanas del bloque (SEP, 2011b). Los temas y proyectos en el libro de texto se guían por los aprendizajes esperados de la sección; estos son también un referente para la planeación de la clase y la evaluación de los alumnos. 
Bio - grafia. Escritos sobre la Biología y su Enseñanza. ISSN 2027

Edición Extraordinaria. p.p. 621 - 635

Memorias del VIII Encuentro Nacional de Experiencias en Enseñanza de la Biología y la Educación Ambiental. III Congreso Nacional de Investigación en Enseñanza de la Biología.

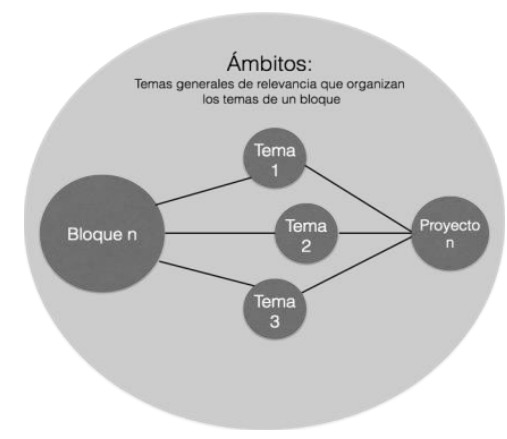

Figura 1. Estructura de un bloque en ciencias naturales.

El trabajo por proyectos (TPP) suele tener relación con la educación ambiental puesto que ésta generalmente sugiere actividades complejas que requieren trabajo colaborativo, investigación, propuestas, desarrollo de acciones, evaluación del impacto, etc. Los proyectos que se plantean en el libro de texto pretenden dar cierre a los bloques al integrar el conocimiento y los aprendizajes esperados de los temas vistos. Una finalidad del trabajo por proyectos es favorecer la apropiación de competencias de los alumnos y las alumnas por medio de la "puesta en práctica" de los conocimientos (SEP, 2011 a: 25) (ver tabla 1). Los proyectos analizados se seleccionaron por presentar temáticas relacionadas con la educación ambiental. La descripción de los dos proyectos analizados se presenta en la Tabla 2. 
Bio - grafia. Escritos sobre la Biología y su Enseñanza. ISSN 2027

Edición Extraordinaria. p.p. 621 - 635

Memorias del VIII Encuentro Nacional de Experiencias en Enseñanza de la Biología y la Educación Ambiental. III Congreso Nacional de Investigación en Enseñanza de la Biología.

\begin{tabular}{|c|c|}
\hline Bloque 2 "¿Cómo somos los seres vivos?" & $\begin{array}{c}\text { Bloque } 3 \text { "¿Cómo transformamos la } \\
\text { naturaleza?" }\end{array}$ \\
\hline Ámbitos: la vida; el ambiente y la salud & Ámbitos: los materiales; la tecnología \\
\hline $\begin{array}{l}\text { Tema } 1 \text { "Cambios en los seres vivos y } \\
\text { procesos de extinción" }\end{array}$ & $\begin{array}{l}\text { Tema } 1 \text { "Relación entre las propiedades de } \\
\text { los materiales y el consumo responsable" }\end{array}$ \\
\hline $\begin{array}{l}\text { Tema } 2 \text { "Importancia de las interacciones } \\
\text { entre los componentes del ambiente" }\end{array}$ & $\begin{array}{l}\text { Tema } 2 \text { "Importancia de las } \\
\text { transformaciones temporales y } \\
\text { permanentes de los materiales" }\end{array}$ \\
\hline $\begin{array}{l}\text { Tema } 3 \text { "Relación de la contaminación del } \\
\text { aire con el calentamiento global y el cambio } \\
\text { climático" }\end{array}$ & $\begin{array}{l}\text { Tema } 3 \text { "Aprovechamiento e identificación } \\
\text { del funcionamiento de las maquinas simples" }\end{array}$ \\
\hline Proyecto 2 "Mejoremos nuestro ambiente" & $\begin{array}{l}\text { Proyecto } 3 \text { "Reúso y reciclado de } \\
\text { materiales" }\end{array}$ \\
\hline
\end{tabular}

Tabla 1. Contenido de bloques dos y tres del libro texto de ciencias naturales donde se encuentran los proyectos seleccionados para éste estudio. 
Bio - grafia. Escritos sobre la Biología y su Enseñanza. ISSN 2027

Edición Extraordinaria. p.p. 621 - 635

Memorias del VIII Encuentro Nacional de Experiencias en Enseñanza de la Biología y la Educación Ambiental. III Congreso Nacional de Investigación en Enseñanza de la Biología.

\begin{tabular}{|c|c|}
\hline $\begin{array}{l}\text { Proyecto dos: "Mejoremos nuestro } \\
\text { ambiente" }\end{array}$ & $\begin{array}{l}\text { El proyecto tres: "Reúso y reciclado de } \\
\text { materiales" }\end{array}$ \\
\hline $\begin{array}{l}\text { Tipo: Proyecto de tipo ciudadano, partiendo } \\
\text { de una clasificación señalada en los } \\
\text { documentos (ciudadano, investigación o } \\
\text { tecnológico). } \\
\text { Autonomía del/la alumno(a): La decisión del } \\
\text { tema específico a analizar en este proyecto } \\
\text { la deben de tomar los estudiantes con el } \\
\text { apoyo del docente. Propone preguntas como } \\
\text { ¿Qué es huella ecológica? ¿Qué acciones } \\
\text { pueden realizarse para conservar la } \\
\text { naturaleza? ¿Qué acciones perjudiciales }\end{array}$ & $\begin{array}{l}\text { Objetivo: Se centra la búsqueda, } \\
\text { organización y reflexión en torno a } \\
\text { información sobre el reúso y reciclado de } \\
\text { materiales, pero no sugiere llevar estas } \\
\text { actividades a la acción comunitaria. } \\
\text { Organización: tres fases: planeación, } \\
\text { desarrollo y comunicación. } \\
\text { Tipo: proyecto de carácter científico que } \\
\text { puede extenderse a ser tecnológico, pues se } \\
\text { propone la elaboración de una máquina }\end{array}$ \\
\hline
\end{tabular}


Bio - grafia. Escritos sobre la Biología y su Enseñanza. ISSN 2027

Edición Extraordinaria. p.p. 621 - 635

Memorias del VIII Encuentro Nacional de Experiencias en Enseñanza de la Biología y la Educación Ambiental. III Congreso Nacional de Investigación en Enseñanza de la Biología.

para la naturaleza observan en el lugar donde viven?, entre otras.

Tabla 2. Descripción de los proyectos dos y tres del libro de Ciencias Naturales de sexto grado, analizados en este estudio.

Diseño del instrumento

El diseño de la matriz se basó en el marco teórico presentado e ideas extraídas del trabajo de Rentería-Guzmán (2007). Esta matriz se caracteriza por tener tres niveles de complejidad compuestos por varias categorías u observables que los definen. Las observables y su descripción facilitan la identificación de los contenidos ambientales para el análisis posterior (ver Tabla 3). 
Bio - grafia. Escritos sobre la Biología y su Enseñanza. ISSN 2027

Edición Extraordinaria. p.p. 621 - 635

Memorias del VIII Encuentro Nacional de Experiencias en Enseñanza de la Biología y la Educación Ambiental. III Congreso Nacional de Investigación en Enseñanza de la Biología.

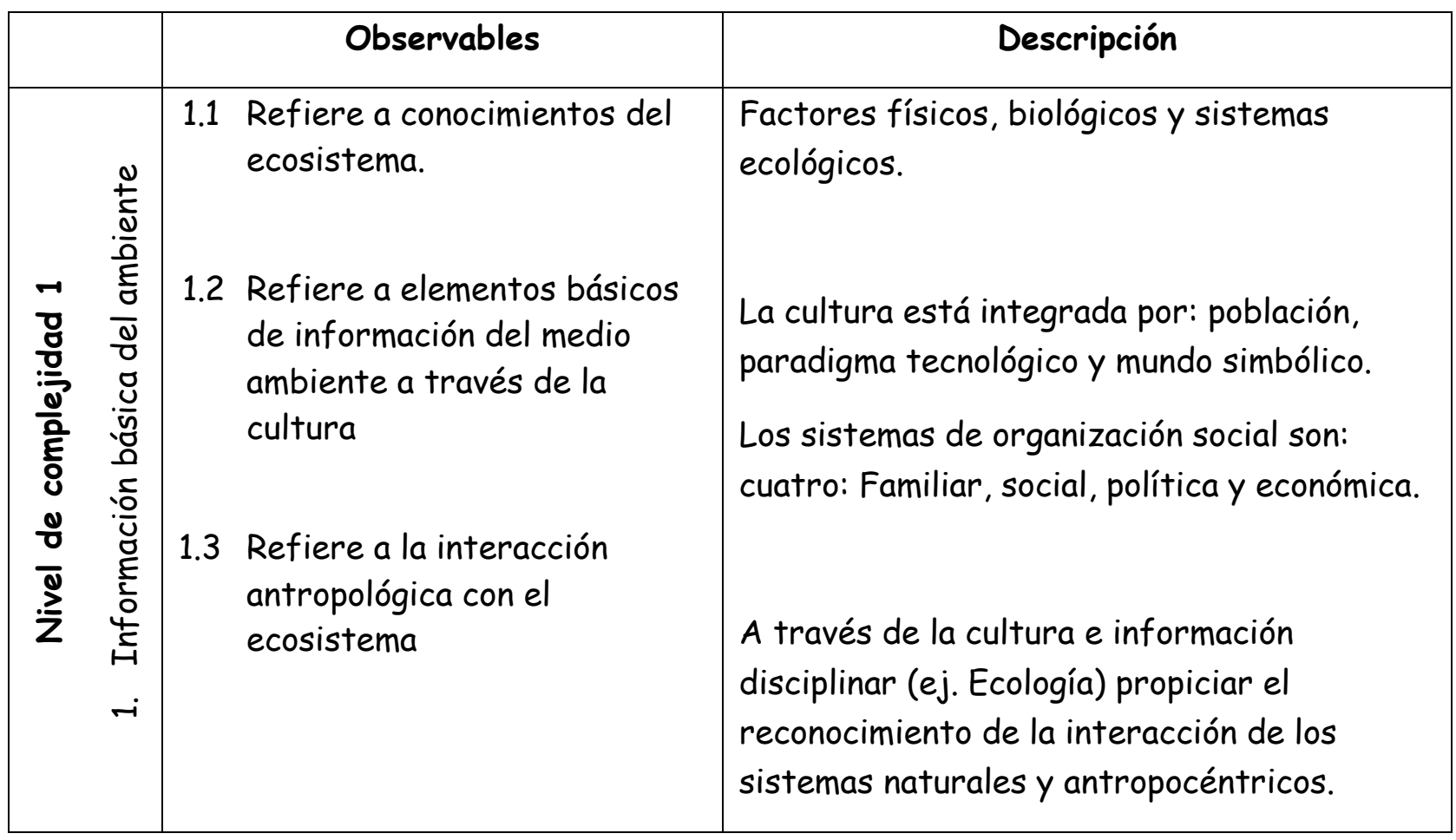


Bio - grafia. Escritos sobre la Biología y su Enseñanza. ISSN 2027

Edición Extraordinaria. p.p. 621 - 635

Memorias del VIII Encuentro Nacional de Experiencias en Enseñanza de la Biología y la Educación Ambiental. III Congreso Nacional de Investigación en Enseñanza de la Biología.

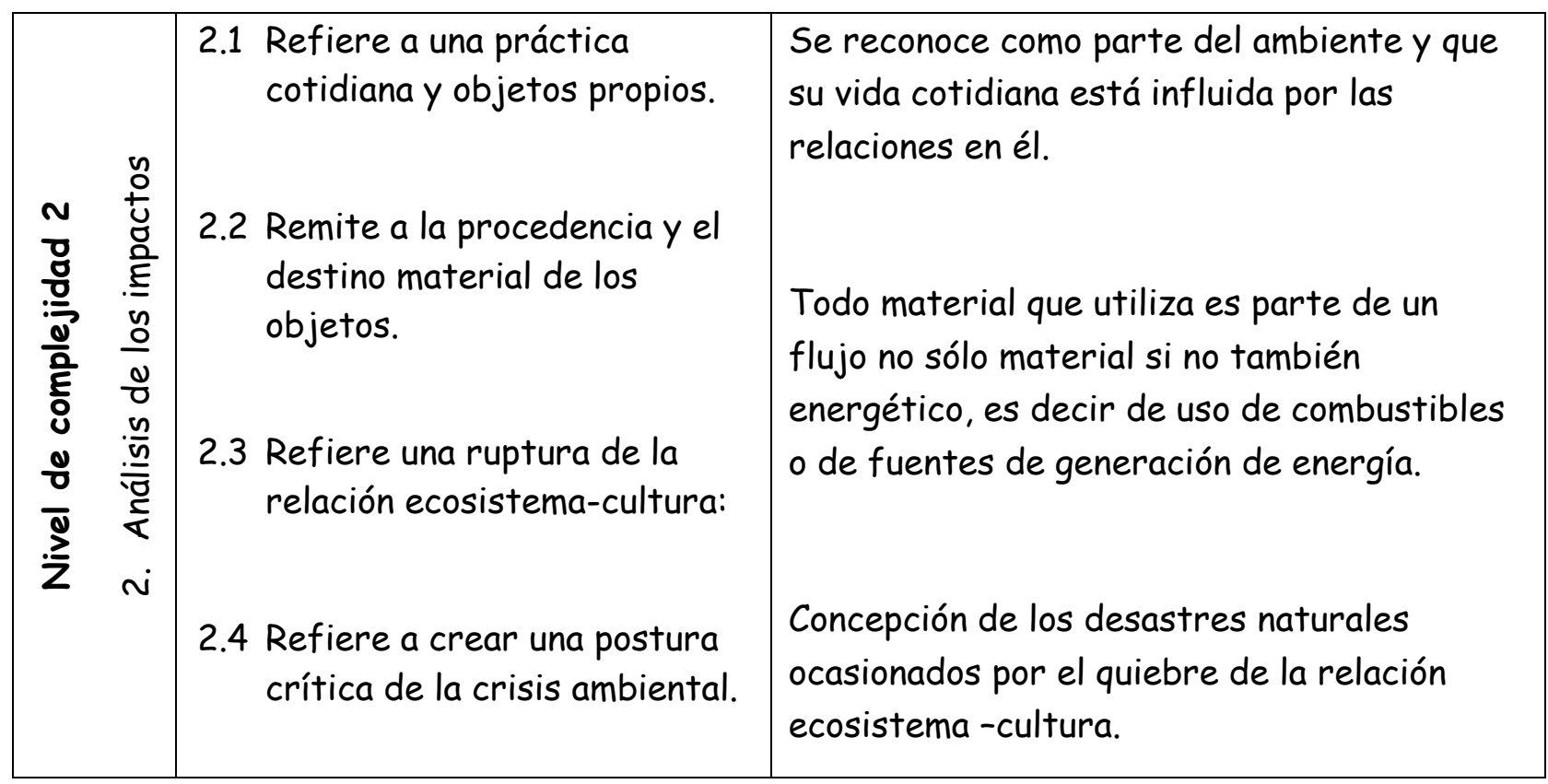


Bio - grafia. Escritos sobre la Biología y su Enseñanza. ISSN 2027

Edición Extraordinaria. p.p. 621 - 635

Memorias del VIII Encuentro Nacional de Experiencias en Enseñanza de la Biología y la Educación Ambiental. III Congreso Nacional de Investigación en Enseñanza de la Biología.

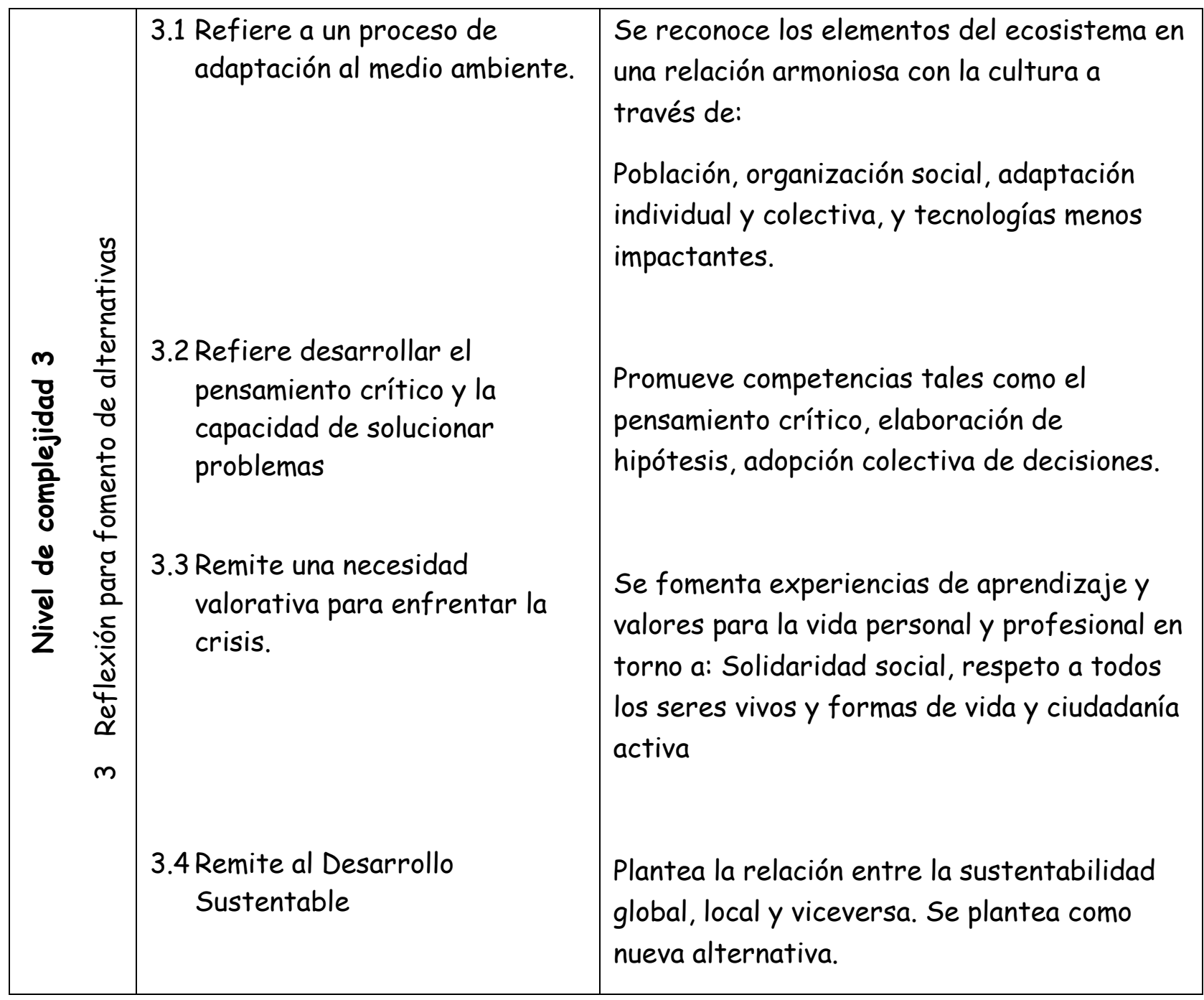

Tabla 3. Matriz de evaluación para contenidos ambientales. 
Bio - grafia. Escritos sobre la Biología y su Enseñanza. ISSN 2027

Edición Extraordinaria. p.p. 621 - 635

Memorias del VIII Encuentro Nacional de Experiencias en Enseñanza de la Biología y la Educación Ambiental. III Congreso Nacional de Investigación en Enseñanza de la Biología.

\begin{abstract}
ANÁLISIS
Empleamos el análisis cualitativo del contenido, esta estrategia permitió acotar el enfoque por medio de categorías y visualizar relaciones entre los elementos que las componen (Schreier, 2012). Para aumentar la validez cada autora aplicó la matriz diseñada para codificar, posteriormente reunimos los resultados y discutimos los hallazgos.
\end{abstract}

\title{
RESULTADOS
}

Primer nivel de complejidad: Información básica del medio ambiente. Identificamos algunos contenidos ambientales básicos en ambos proyectos. Inclusive, hay temas que no se cubren en el cuerpo del bloque, pero que dan mayor complejidad a los temas vistos, por ejemplo: "La huella ecológica se relaciona con la cantidad de recursos que utilizas en tu vida cotidiana..." (SEP, 2011b:78, Proyecto 2) o "Hay otro tipo de contaminación...acústica o por ruidos" (SEP, 2011b:106, Proyecto 3). Los proyectos sugieren a los alumnos y las alumnas la búsqueda de información sobre elementos básicos del ecosistema y del ambiente. Aunque, son las competencias de los docentes y de los estudiantes de las que depende el éxito potencial de estos contenidos en la práctica.

Segundo nivel de complejidad: Análisis de los impactos. Los proyectos tienen algunos elementos que apuntan a que el/la alumno(a) practique la reflexión crítica sobre temas ambientales. El contenido apunta a una visión conservacionista "¿Qué acciones se pueden realizar para conservar el ambiente?" (SEP, 2011b:79, Proyecto 2) y tiene poco énfasis en el pensamiento sistémico que busca una visión crítica de los temas ambientales. Sin embargo la reflexión acerca de la ruptura ecosistema-cultura y los impactos antropológicos en el ambiente son introducidos por medio de preguntas o temas de investigación: "Realizaran investigaciones con el reúso y el reciclado de los materiales...la posible aplicación de la tecnología para solucionar problemas de deterioro ambiental..." (SEP, 2011b:106, Proyecto 3). A través de breves notas se señalan ideas en torno a los factores causales de las problemáticas como "Es común que muchos de nosotros...utilicemos recursos indiscriminadamente, sin saber siquiera de dónde proceden" (SEP, 2011b:78, Proyecto 2), 
Bio - grafia. Escritos sobre la Biología y su Enseñanza. ISSN 2027

Edición Extraordinaria. p.p. 621 - 635

Memorias del VIII Encuentro Nacional de Experiencias en Enseñanza de la Biología y la Educación Ambiental. III Congreso Nacional de Investigación en Enseñanza de la Biología.

pero no se trata ésta complejidad desde la transversalidad, puesto que el contenido de los proyectos se limita a las ciencias naturales.

Tercer nivel de complejidad: Reflexión para fomento de alternativas. Identificamos evidencia de fomento hacia un sistema de valores al aprecio de la naturaleza y el uso racional de los recursos naturales. Se indican niveles de responsabilidad personal al impulsar al/a la alumno(a) a pensar en el impacto de su consumo y el impacto del mismo en el ambiente: "Cada uno de nosotros puede realizar actividades sencillas que contribuyan a disminuir la generación de gases y ahorrar energía..." (SEP, 2011b:78, Proyecto 2). No obstante, no encontramos acciones de intervención comunitaria ni evidencia de transversalidad en estos proyectos que conecten dilemas éticos, formación y responsabilidad ciudadana, gestión ambiental u otro tipo de áreas que complementan las necesidades de un esquema ambiental gestionado con responsabilidad y respeto.

\section{DISCUSIÓN Y CONCLUSIONES}

Los contenidos ambientales de los proyectos seleccionados en relación a los aprendizajes esperados del currículo de ciencias naturales para sexto grado son insuficientes. El aprendizaje esperado del bloque 2 señala la necesidad de analizar causas y efectos a la salud de la contaminación ambiental y temas relacionados con el calentamiento global aspectos que no se retoman en el TPP.

De igual manera en el bloque 3 los aprendizajes señalan la necesidad de fomentar el consumo responsable y la reducción de desechos, observándose que en el TPP solo se promueve la separación y reciclado de materiales sin profundizar la problemática principal de los desechos.

Aunque los contenidos ambientales manejados en estas unidades son pertinentes con las problemáticas actuales de la educación ambiental para la sustentabilidad existe una falta de complejidad en los contenidos de los bloques 2 y 3 del libro de texto de ciencias naturales para sexto grado ya que cubren los temas con generalidad y poca transversalidad. Aunque, son las competencias de los docentes y de los estudiantes las que depende el éxito 
Bio - grafia. Escritos sobre la Biología y su Enseñanza. ISSN 2027

Edición Extraordinaria. p.p. 621 - 635

Memorias del VIII Encuentro Nacional de Experiencias en Enseñanza de la Biología y la Educación Ambiental. III Congreso Nacional de Investigación en Enseñanza de la Biología.

potencial de estos contenidos en la práctica, concordando lo anterior con el trabajo de Parga, et al. 2013

La educación ambiental se ha relacionado en mayor proporción con el estudio de las ciencias. En este caso, sugerimos que los proyectos ambientales sean planeados de manera transversal con las otras asignaturas y quizá desarrollar un proyecto por ciclo para alcanzar mayor grado de complejidad en el tratamiento de los temas. El empleo de la matriz aquí desarrollada puede ayudar a detectar los niveles de complejidad planteados en libros de texto y abonar a desarrollar una visión sistémica de los contenidos incorporando diversos niveles de complejidad, sin embargo esta matriz de análisis debe ser complementada al aplicarla a otros proyectos y con la participación de maestros y alumno(a)s.

\section{REFERENCIAS}

Benítez Esquivel, N. (2009). "Nuevos contenidos para la educación ambiental". En Educación ambiental en la formación docente en México. Meixueiro Hernández, A., Ramírez Beltran, R. y Ruiz Cruz, J. (Coords) Universidad Pedagógica Nacional, México D.F. Págs. 39-59.

Maya, A. (1996). El reto de la vida. Ecosistema y cultura, una introducción al estudio del medio ambiente. Ecofondo. Colombia.

Peza Hernandez, G. (2014). Educación ambiental para la sustentabilidad. En Silva Mar, M., Huerta Chúa, A., Ruiz Carús, S., et al. (Eds.). "Educación ambiental para la sustentabilidad". Grupo Editorial Cenzontle, México, D.F. p. 88-95.

Renteria Guzmán, Y. (2007). Analisis de la incorporación de propuestas educativas constructivistas y ambientales a la educación básica. El ejemplo del eje ambiental del manual de transversalidad. Tesis de licenciatura. Universidad de Guadalajara, México. (no publicada aún).

Schreier, M. 2012. Qualitative Content Analysis in Practice, London, U.K., SAGE Publications LId. 
Bio - grafia. Escritos sobre la Biología y su Enseñanza. ISSN 2027

Edición Extraordinaria. p.p. 621 - 635

Memorias del VIII Encuentro Nacional de Experiencias en Enseñanza de la Biología y la Educación Ambiental. III Congreso Nacional de Investigación en Enseñanza de la Biología.

Secretaría de Medio Ambiente y Recursos Naturales. SEMARNAT. (2006). Estrategia de educación ambiental para la sustentabilidad en México (versión ejecutiva): Estrategia nacional 2006-2014. México: SEMARNAT

Secretaría de Educación Pública. SEP. (2011a). Programas de Estudio 2011. Educación Básica Primaria. Sexto Grado. México, D.F.

Secretaría de Educación Pública. SEP. (2011b). Ciencias Naturales. Sexto Grado. 2 da. Edición. 3era. edición revisada, ciclo escolar 2014-2015. México, D.F.

Parga Lozano, D. Mora W. y Cardenas Y. (2013). La dimensión ambiental: "una inclusión necesaria en la formación de profesores de química". Bio-grafía Escritos sobre la Biología y su Enseñanza. Edición Extra-Ordinaria. Memorias del VII Encuentro Nacional de Experiencias en la Enseñanza de la Biología y la Educación Ambiental y II Congreso Nacional de Investigación en la Enseñanza de la Biología. 